\title{
NOTE ON STRONGLY REGULAR NEAR-RINGS
}

\author{
by MOTOSHI HONGAN
}

(Received 29th May 1985)

Let $S$ be a semigroup. An element $a$ of $S$ is called right (resp. left) regular if $a=a^{2} x$ (resp. $a=x a^{2}$ ) for some $x \in S$. If $a$ is regular and right (resp. left) regular, $a$ is called strongly right (resp. left) regular. As is well known, if $a$ is strongly regular (i.e., right and left regular) then it is regular, more precisely, there exists uniquely an element $x$ such that $a=a^{2} x, x=x^{2} a$ and $a x=x a$, and $a$ is contained in a subgroup of $S$ (and conversely).

Following M. Petrich [2], a semigroup $S$ is called weakly commutative if for each pair of elements $x, y$ in $S$ there exists a positive integer $m$ and an element $z$ in $S$ such that $(x y)^{m}=y z x$. Let $a$ be an element of a weakly commutative semigroup $S$. Then $a$ is strongly regular if (and only if) $a \in\langle a\rangle^{2}$, where $\langle a\rangle$ is the ideal of $S$ generated by $a$, or equivalently, $a=$ uavaw for some $u, v, w \in S^{1}$, the semigroup obtained from $S$ by adjoining an identity. Actually, there exist positive integers $k, l$ and $x, y \in S^{1}$ such that $(u a v)^{k}=a v x u$ and $\left(v x u a w^{k}\right)^{l}=a w^{k} y v x u$. Hence $a=u a v a w=(u a v)^{k} a w^{k}=a v x u a w^{k}=a\left(v x u a w^{k}\right)^{l}=a^{2} w^{k} y v x u$; similarly, $a$ is left regular.

An element $a$ of $S$ is called $\pi$-regular if there exists a positive integer $n$ such that $a^{n}$ is regular, and right (resp. left) $\pi$-regular if there exists a positive integer $n$ such that $a^{n}$ is right (resp. left) regular; $a$ is strongly $\pi$-regular if $a$ is both right and left $\pi$-regular. And, $a$ is called strongly right (resp. left) $\pi$-regular if there exists a positive integer $n$ such that $a^{n}$ is strongly right (resp. left) regular, namely, $a^{n}=a^{2 n} x a^{n}$ (resp. $a^{n}=a^{n} x a^{2 n}$ ) for some $x \in S$. The semigroup $S$ is called $\pi$-regular if every elements of $S$ is $\pi$-regular, and right (resp. left) $\pi$-regular if every element of $S$ is right (resp. left) $\pi$-regular; $S$ is strongly $\pi$ regular if every element of $S$ is strongly $\pi$-regular. Similarly, $S$ is called strongly right (resp. left) $\pi$-regular if every element of $S$ is strongly right (resp. left) $\pi$-regular. As is easily seen, $S$ is strongly right (resp. left) $\pi$-regular if and only if $S$ is $\pi$-regular and right (resp. left) $\pi$-regular.

In view of [2, Theorem IV.1.6] (see also Theorem 2 below), every strongly right (or left) regular semigroup is strongly regular. As an application of this result, we shall prove the following which includes [3, Theorem 12 and Proposition 13]:

Theorem 1. Let $N$ be a (left) near-ring. Then the following are equivalent:

(1) $N$ is strongly regular.

(2) $N$ is right regular.

(3) $N$ is left regular and right $\pi$-regular. 
(4) $N$ is strongly right regular.

(5) $N$ is strongly left regular.

(6) $N$ is regular and ae=eae for any idempotent $e$ and any element $a$ in $N$.

In advance of proving Theorem 1, we state the following:

Lemma 1. Let $N$ be a right (resp. left) regular near-ring, and let $a$ and $b$ be elements of $N$.

(1) If $a b=0$ then $b a=0 a$.

(2) If $a b=0$ and $b^{n}=0 b$ for some $n>1$, then $b=0$. In particular, $N$ contains no non-zero nilpotent element.

Proof. (1) There exists $x \in N$ such that $b a=(b a)^{2} x=0 a x$ (resp. $b a=x(b a)^{2}=0 a$ ). Then $0 a=a b a=a 0 a x=0 a x=b a$.

(2) There exists $y \in N$ such that $b=b^{2} y=b^{n} y^{n-1}=0 b y^{n-1} \quad$ (resp. $b=y b^{2}=y^{n-1} b^{n}=$ $y^{n-1} 0 b=0 b$ ). Then $0 \mathrm{~b}=b^{n}=b^{n-1} 0 b y^{n-1}=0 b y^{n-1}=b$, and so $b=0 b=a 0 b=a b=0$.

Proof of Theorem 1. Obviously, (1) implies (2)-(6) (see Lemma 1 (2)), and [2, Theorem IV.1.6] shows that (1), (4) and (5) are equivalent.

$(3) \Rightarrow(2)$. Let $a$ be an arbitrary element of $N$. Then there exists a positive integer $n$ and $x \in N$ such that $a^{n}=a^{n+1} x$. If $n>1$ then $a^{n-1}\left(a^{n-1}-a^{n} x\right)=0$. By Lemma $1(1),\left(a^{n-1}-\right.$ $\left.a^{n} x\right) a^{n-1}=0 a^{n-1}$ and $\left(a^{n-1}-a^{n} x\right) a^{n} x=0 a^{n} x$, and hence $\left(a^{n-1}-a^{n} x\right)^{2}=0\left(a^{n-1}-a^{n} x\right)$. Then, Lemma 1 (2) proves that $a^{n-1}=a^{n} x$. Continuing this procedure, we obtain eventually $a=a^{2} x$.

$(2) \Rightarrow(4)$. Let $a$ be an arbitrary element of $N$, and $a=a^{2} x$. Since $a(a-a x a)=0=$ $a \times a(a-a \times a)$, we have $(a-a \times a) a=0 a$ and $(a-a \times a) a x a=0 a x a$ (Lemma 1 (1)), and hence $(a-a \times a)^{2}=0(a-a x a)$. Then Lemma 1 (2) shows that $a=a x a$.

$(6) \Rightarrow(2)$. Given $a \in N$, there exists $x \in N$ such that $a=a x a$. Note that $a x$ and $x a$ are idempotents. Then, by (6), we have $a=a x a=a(x \cdot a x) a=a(a x \cdot x \cdot a x) a=a^{2}\left(x^{2} a x\right) a$.

Remark. In [3], a near-ring $N$ is called right (resp. left) regular if for every $a$ there is an $x$ in $N$ such that $a=a^{2} x$ (resp. $a=x a^{2}$ ) and $a=a x a$, and $N$ is called right (resp. left) strongly regular if $N$ is right (resp. left) regular in our sense. Obviously, if $N$ is right (resp. left) regular in the sense of [3] then it is strongly right (resp. left) regular.

In view of a theorem of Zöschinger-Dischinger (see, e.g., [1, Proposition 2]), every right (or left) $\pi$-regular ring is strongly $\pi$-regular. It seems difficult to extend this result to semigroups without any restriction. We shall give the following generalization of [2, Theorem IV.1.6.].

Theorem 2. A semigroup $S$ is strongly $\pi$-regular if it is strongly right (or left) $\pi$ regular.

Proof. It suffices to show that if $a=a^{2} x a$ then $a$ is left regular. There exists a positive 
integer $n$ and $y \in S$ such that $(a x)^{n}=(a x)^{2 n} y(a x)^{n}$. Then $a=a \cdot a \cdot x a=a^{n} \cdot a \cdot(x a)^{n}=$ $a^{n}(a x)^{n} a=a^{n}(a x)^{2 n} y(a x)^{n} a=\left\{a^{n}(a x)^{n} a\right\} x(a x)^{n-1} y(a x)^{n} a=a x(a x)^{n-1} y(a x)^{n} a=(a x)^{n} y(a x)^{n} a$ $=(a x)^{2 n} y\left\{(a x)^{n} y(a x)^{n} a\right\}=(a x)^{2 n} y a$. Since $a x=a(a x)^{2}=a^{2 n-1}(a x)^{2 n}$, we see that $a=$ $(a x)^{2 n-1} \cdot a x \cdot y a=(a x)^{2 n-1} a^{2 n-1}\left\{(a x)^{2 n} y a\right\}=(a x)^{2 n-1} a^{2 n}$.

Corollary 1. Let $S$ be a subsemigroup of a left (resp. right) $\pi$-regular semigroup $T$. If $S$ is right (resp. left) $\pi$-regular, then it is strongly $\pi$-regular.

Proof. Given $a \in S$, there exists a positive integer $n, s \in S$ and $t \in T$ such that $a^{2 n} s=$ $a^{n}=t a^{2 n}$. Since $a^{n} s=t a^{2 n} s=t a^{n}$, we see that $a^{n}=t a^{2 n}=a^{n} s a^{n}$. Hence, $S$ is strongly $\pi$-regular, by Theorem 2 .

\section{REFERENCES}

1. Y. Hirano, Some studies on strongly $\pi$-regular rings, Math. J. Okayama Univ. 20 (1978), 141149.

2. M. Petrich, Introduction to semigroups (Merrill, Columbus, Ohio, 1973).

3. Y. V. Reddy and C. V. L. N. Murty, On strongly regular near-rings, Proc. Edinburgh Math. Soc. 27 (1984), 61-64.

Tsuyama College of Technology

Numa, Tsuyama, Okayama

JAPAN 